\title{
List of Charts
}

Chart 3.1: Maasai notions of purity associated with age and sex

Chart 3.2: Ranked scales of status among the Maasai and Hindus

Chart 6.1: Evidence of brainwashing in Samburu ilmugit ceremonies

Chart 8.1: Episodes of Chamus oral history compared with evolutionary processes 